\title{
Adaptive Handover Decision Algorithm Based on Multi-Influence Factors through Carrier Aggregation Implementation in LTE-Advanced System
}

\author{
Ibraheem Shayea, ${ }^{1}$ Mahamod Ismail, ${ }^{1}$ Rosdiadee Nordin, ${ }^{1}$ and Hafizal Mohamad ${ }^{2}$ \\ ${ }^{1}$ Faculty of Engineering and Built Environment, Universiti Kebangsaan Malaysia, 43600 Bangi, Selangor, Malaysia \\ ${ }^{2}$ MIMOS Berhad, Technology Park Malaysia, 57000 Kuala Lumpur, Malaysia \\ Correspondence should be addressed to Ibraheem Shayea; shaia2009@yahoo.com
}

Received 30 May 2014; Accepted 6 November 2014; Published 26 November 2014

Academic Editor: Peter Mueller

Copyright (C) 2014 Ibraheem Shayea et al. This is an open access article distributed under the Creative Commons Attribution License, which permits unrestricted use, distribution, and reproduction in any medium, provided the original work is properly cited.

\begin{abstract}
Although Long Term Evolution Advanced (LTE-Advanced) system has benefited from Carrier Aggregation (CA) technology, the advent of CA technology has increased handover scenario probability through user mobility. That leads to a user's throughput degradation and its outage probability. Therefore, a handover decision algorithm must be designed properly in order to contribute effectively for reducing this phenomenon. In this paper, Multi-Influence Factors for Adaptive Handover Decision Algorithm (MIFAHODA) have been proposed through CA implementation in LTE-Advanced system. MIF-AHODA adaptively makes handover decisions based on different decision algorithms, which are selected based on the handover scenario type and resource availability. Simulation results show that MIF-AHODA enhances system performance better than the other considered algorithms from the literature by $8.3 \mathrm{~dB}, 46 \%$, and $51 \%$ as average gains over all the considered algorithms in terms of SINR, cell-edge spectral efficiency, and outage probability reduction, respectively.
\end{abstract}

\section{Introduction}

In mobile wireless systems, there are several handover decision algorithms (HODAs) which have been proposed based on different parameters such as (i) Received Signal Strength (RSS), (ii) RSS with a threshold, (iii) RSS with hysteresis, (iv) RSS with hysteresis and threshold (parameters (i) to (iv) are discussed in detail from Pollini) [1], (v) RSS with hysteresis and distance [2], (vi) Signal-to-Interference-plus-NoiseRatio (SINR) [3], and (vii) Interference-to-Interference-plusNoise-Ratio (IINR) [4]. All of these HODAs have been proposed for the purpose of taking an intact handover decision in order to enhance system performance through the user's mobility. However, in $[1,3,4]$, all the HODAs are taken based on a single parameter, while there are other influencing factors which have not been considered. That leads to taking nonintact handover decisions, which in turn degrades a user's throughput and increases its outage probability. Thus, the communication efficiency between the user and serving network is negatively affected. In [2], HODA is taken based on multiple factors, but there are other influencing factors that have not been considered such as the interferences, noise, and resource availability. These effectively impact system performance. Furthermore, the advent of CA technology has added a new handover scenario, which can be performed between the serving component carriers (CCs) under the same sector and the same evolved node $\mathrm{B}$ (eNB) to change the primary component carriers (PCCs). This leads to increased handover probability, which in turn leads to increased throughput degradation and user outage probability. This type of handover scenario can be reduced as long as the serving PCC provides acceptable RSS to the served user equipment (UE). Therefore, more efficient HODA is needed, which should contribute for reducing user throughput degradation and high outage probability.

In this paper, MIF-AHODA has been proposed in order to provide a seamless handover process through CA implementation in LTE-Advanced system. MIF-AHODA is automatically 
changing handover decision algorithm based on the handover scenario type and availability of resources. This algorithm aims to enhance system performance in the perspective of SINR, cell-edge spectral efficiency, and outage probability reduction through the users' mobility.

The remainder of this paper is organized as follows: Related Work is described in Section 2 followed by Handover with CA Technique in Section 3 and then Proposed Algorithm in Section 4. Next, System Model is described in Section 5 and then Results and Discussions in Section 6 and Conclusion in Section 7.

\section{Related Work}

HODA is an essential step of the handover procedure in cellular wireless networks. It should be designed carefully in order to take an intact and a proper handover decision to the suitable target cell. That provides a seamless connection between the UE and serving eNB through its roaming within the cells. Anyway, handover decision is taken by the serving eNB based on the measurement report (MR) that is received from the served UE. MR contains the signals levels list of specific neighbor cells, and it can contain other information based on the implemented HODA. However, there are several HODAs that have been proposed [1-4] based on different parameters, such as HODA based on RSS [1], RSS and distance [2], SINR [3], and IINR [4] with considering the hysteresis level. All these HODAs aim to enhance system performance through the user's mobility within the cells.

In [1], handover decision algorithm is proposed to be taken based on the Received Signal Strength (HODA-RSS). The algorithm triggers handover once the target RSS $\left(\mathrm{RSS}_{T}\right)$ level becomes sufficiently stronger than the serving RSS $\left(\mathrm{RSS}_{S}\right)$ by a handover margin level $\left(M_{\mathrm{RSS}}\right)$ in $\mathrm{dB}$. That algorithm can be simplified by

$$
\mathrm{RSS}_{T}>\mathrm{RSS}_{S}+M_{\mathrm{RSS}}
$$

In [2], handover decision algorithm based on distance and relative Received Signal Strength (HODA-D-RSS) has been proposed in a log-normal fading environment. The handover decision output becomes true and starts for initiating handover procedure once the two following conditions are met; (i) the measured distance between user and target eNB becomes less than that between the user and target eNB by a certain threshold distance and (ii) the average target RSS becomes stronger than that received from the serving eNB by a given hysteresis level. That HODA can be simplified by the following:

$$
\begin{aligned}
& \operatorname{Dis}_{T}<\mathrm{Dis}_{S}+\gamma_{d}, \\
& \mathrm{RSS}_{T}>\mathrm{RSS}_{S}+M_{\mathrm{RSS}},
\end{aligned}
$$

where $\mathrm{Dis}_{T}$ and $\mathrm{Dis}_{S}$ represent the distance from the user to the target and serving eNBs, respectively, while $\gamma_{d}$ is the distance margin level.

In [3], handover decision algorithm has been designed utilizing SINR (HODA-SINR) as control handover parameters for taking the handover decision. The algorithm allows the served user to trigger the handover once the target SINR quality $\left(\operatorname{SINR}_{T}\right)$ becomes sufficiently better than the serving SINR quality $\left(\operatorname{SINR}_{S}\right)$ by a certain hysteresis margin level $\left(M_{\text {SINR }}\right)$. For simplicity, this algorithm can be represented by

$$
\mathrm{SINR}_{T}>\mathrm{SINR}_{\mathrm{S}}+M_{\mathrm{SINR}}
$$

where SINR $_{T}$ and $\mathrm{SINR}_{S}$ represent the SINR of target and serving cells, respectively, while $M_{\mathrm{SINR}}$ represents the hysteresis SINR margin level in $\mathrm{dB}$.

In [4], an optimal handover decision algorithm is proposed based on Interference to other- Interferences-plusNoise Ratio (IINR) parameter (HODA-IINR). It is designed from the perspective of throughput enhancement by considering two handover schemes (Fast Cell Selection (FCS) and Soft Handover (SHO)). In case of considering FCS the proposed HODA is represented by $\rightarrow \mathrm{SINR}_{o}-\mathrm{IINR}_{i}<-1$, where SINR $_{o}$ represents SINR from the serving eNB, while $\mathrm{IINR}_{i}$ represents IINR from the target eNB. In the other case, when SHO is considered the proposed HODA is represented by $\rightarrow \mathrm{SINR}_{o}-\mathrm{IINR}_{i}<0$. However, that HODA decides to perform handover only when a throughput gain exists.

These four HODAs take the handover decision based on single parameters (i.e., RSS, Distance, SINR, and IINR). So, they cannot give always a proper handover decision, because there are several influence factors that have not been considered, such as channel condition, Rayleigh fading, interferences, noise, and traffic loads. Also, handover scenario should be considered due to the additional scenario that is added by CA technique, which will be explained in the following section. Therefore, a new handover decision algorithm is needed when CA is considered in LTE-Advanced system.

\section{Handover with CA Technique}

The advent of CA technique in LTE-Advanced system increases the number of aggregated CCs that can be deployed at one eNB and assigned to one UE simultaneously. These CCs are classified into two different types. The first one is known as a PCC, while the second type of CCs is called a SCC $[5,6]$.

The PCC is the carrier that is always being active through the active mode operation of UE. It should provide full cell coverage among the active adjacent CCs or provide the best signal quality over all the active CCs $[6,7]$. However, PCC is normally used for exchange control signaling messages and traffic date between a UE and eNB. It is also used for random access procedure and the allocation of the SCC. In addition, Radio Link Failure (RLF) is recorded when the radio link connection over the PCC is failed, and then the Radio Resource Control (RRC) reestablishment procedure is triggered over the PCC too. Also, the Nonaccess Stratum- (NAS-) recovery procedure is triggered if the RCC reestablishment procedure over the PCC is failed within T310 (T310 is the maximum allowed time for recovering connection through the RRC reestablishment procedure) period of time $[5,8]$.

The UE in LTE-Advanced system release 10 and release 11 (rel.10 and rel.11) can be configured with only one CC among the plurality of assigned CCs as a PCC. At the beginning, 


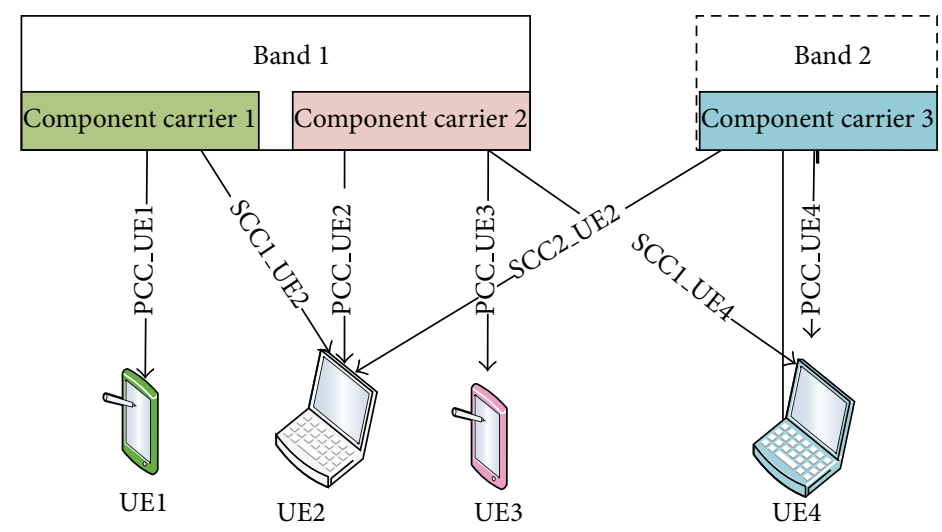

Figure 1: Configuration of CCs for different UEs served by the same eNB.

when the UE sets up the connection to the serving network the PCC is automatically selected by the serving eNB. If only one CC is assigned to the UE, it is configured as a PCC. Otherwise, when several CCs are paired to one UE, one CC among the plural active carriers must be configured as a PCC, while the rest of active CCs should be configured as SCCs [9]. In addition, the configured PCC may be selected from fully configured CCs, rather than being fixed to a particular CC [5]. The selected PCC can differ between UEs which are served by the same eNB. In other words, one CC (i.e., CC1) can be configured as a PCC for UE1 and configured as a SCC for UE2 as illustrated in Figure 1 [8].

The SCC is an additional component carrier that can be configured and activated by eNB when the UE requests a wider bandwidth in order to provide higher data rate to the served UE. In other words, SCC is an additional component carrier which is used for providing additional resources to the served UE, while it cannot be used for exchange control signaling messages between a UE and eNB. However, SCC can be activated or deactivated according to especial conditions, which can be specified according to the UE's request or according to the instructions of the eNB [5].

Implementing CA technique in LTE-Advanced system adds an additional handover scenario, which can occur between component carriers in the same sector, from PCC (CC1) to SCC (CC2) or from PCC (CC2) to SCC (CC1). In other words, the PCC may be switched from $\mathrm{CCl}$ to CC2 or from CC2 to CC1 to change the PCC. So LTEAdvanced system differs than LTE (rel.8 and rel.9), where in LTE system (rel.8 and rel.9) handover occurs between eNBs in different cells or between different sectors under the same eNB only. However, changing the PCC is subjected to several considerations such as looking for the best signal quality or balancing loads between adjacent cells. Switching the CC from PCC to SCC and vice versa is achieved by performing a handover procedure from the PCC (i.e., CC1) to the SCC (i.e., CC2). The handover procedure is performed by UE from the served PCC to the target PCC (which is the SCC) under the same eNB [8].

Consequently, the number of handover scenarios can be increased by implementing CA technique. Thus, there are five handover scenarios that can occur in LTE-Advanced system when CA technology is implemented, which are described in Figure 2 and can be introduced by (i) interfrequency intrasector and intra-eNB handover, (ii) intrafrequency intersector and intra-eNB handover, (iii) interfrequency intersector and intra-eNB handover, (iv) intrafrequency inter-eNB handover, and (v) interfrequency inter-eNB handover [6]. All these handover scenarios are considered in this paper.

Intrafrequency means that the target and the serving carrier frequencies are the same, while interfrequency means that the target and serving carrier frequencies are differentiated from each other. Intrasector means that the target and serving sectors are the same and intersector means that the target and serving sectors are differentiated from each other. Intra-eNB means that the target and serving eNBs are the same, and inter-eNB means that the target and serving eNBs are differentiated from each other.

Increasing handover scenarios leads to increasing the handover probability, which is undesired to users since it leads to increasing the throughput degradation and outage probability. Therefore, an optimal handover decision is requested to reduce the handover probability in order to decrease throughput degradation and outage probability.

\section{Proposed Algorithm}

In this paper, MIF-AHODA based on SINR with handover hysteresis, threshold, and resource availability has been proposed. MIF-AHODA adaptively makes handover decisions based on different decision algorithms, which are selected based on the handover scenario type and resource availability as illustrated in Figure 3. If the handover scenario type is targeting changing the PCC, the handover decision can be taken based on the SINR with handover hysteresis $(M)$ and threshold $(\gamma)$ levels as illustrated in Figure 4(a). Thus, the handover decision algorithm can be expressed as follows:

$$
\begin{aligned}
& S_{S_{-} \mathrm{PCC}} \leq M+\gamma, \\
& S_{T} \geq S_{S_{\text {PPCC }}}+M,
\end{aligned}
$$

where $S_{S \text { PCC }}$ and $S_{T}$ represent the SINR over the serving PCC and target CC, respectively. 


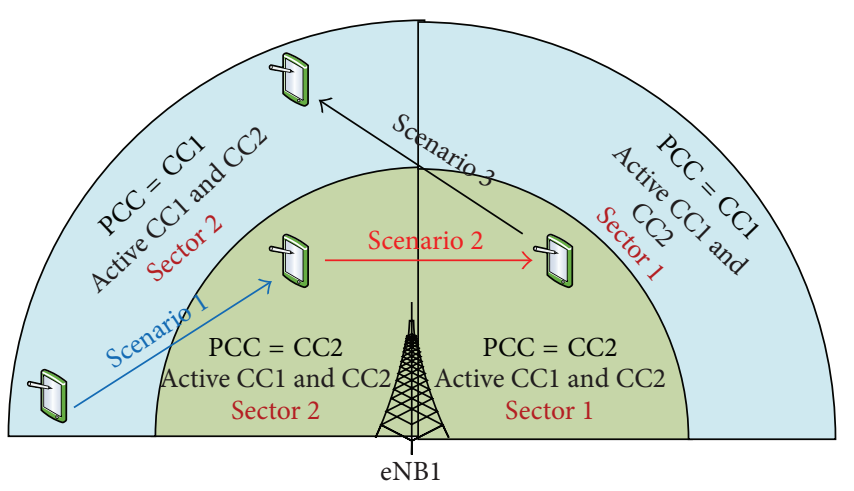

(a) Intra-eNB handover

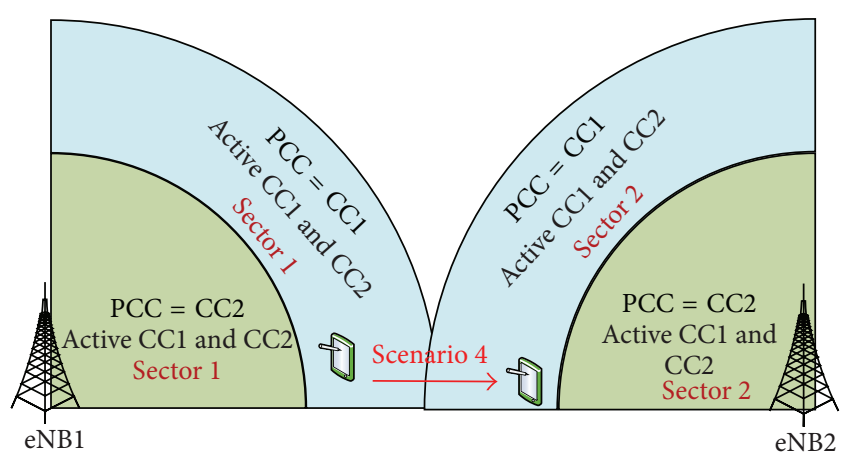

(b) Intrafrequency inter-eNB handover

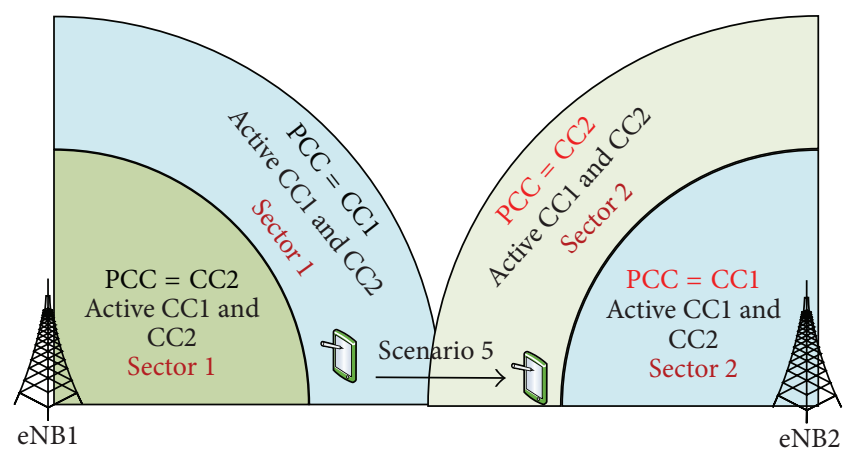

(c) Interfrequency inter-eNB handover

FIgURE 2: Frequency handover scenarios.

On the other hand, if the handover scenario type is targeting changing the serving sector or serving eNB, the handover decision can be adaptively taken based on two different decision algorithms, which are selected based on the resource availability. In the first decision algorithm, if the serving cell has more resources available than the target cell, the handover decision is taken based on the average SINR over both aggregated CCs (PCC and SCC) with handover hysteresis levels as illustrated in Figure 4(b). Also, SINR over the target PCC $\left(S_{T_{-} \text {PCC }}\right)$ should be greater than the threshold $(\gamma)$ level $\left(S_{\text {T_PCC }}>\gamma\right)$. In the second decision algorithm, if the target cell has more resources available than the serving cell by resource Loads Margin level (LM), the handover decision is taken based on the SINR quality over the PCC with hysteresis and threshold levels only, as it is explained in Figure 4(c). Consequently, the handover decision algorithm can be represented by the following expression:

HOD

$$
= \begin{cases}\left(\mathrm{AS}_{T}>\mathrm{AS}_{S}+M\right),\left(S_{T \_ \text {PCC }}>\gamma\right) & \text { if } L_{S} \geq\left(L_{T}+\mathrm{LM}\right) \\ S_{T_{-} \mathrm{PCC}} \geq(M+\gamma) & \text { if } L_{T} \geq\left(L_{S}+\mathrm{LM}\right),\end{cases}
$$

where $\mathrm{AS}_{S}, \mathrm{AS}_{T}$ represent the average SINR over all the aggregated CCs of serving and target eNBs, respectively. $L_{S}$, $L_{T}$ represent the resource Loads availability of serving and target eNBs, respectively. LM is assumed to be $10 \%$ of the average resource Loads availability of the serving and target eNBs.

\section{System Model}

The LTE-Advanced system is modeled based on 3GPP specifications that were introduced in [10]. The network consists of 61 macrohexagonal cell layout models with 500 meter inter-site-distance. One eNB located at the centre of each cell with considering three sectors in each cell and each sector configured with two contiguous CCs. $20 \mathrm{MHz}$ is considered as carrier bandwidth for each CC. Operating frequencies of $\mathrm{CC} 1$ and $\mathrm{CC} 2$ are assumed to be 2 and $2.0203 \mathrm{GHz}$, respectively. The antenna of each CC is pointed toward a different flat side of the hexagonal cell. The transmitted power from all the eNBs for each CC is assumed to be the same. Random numbers of UEs are generated and removed randomly at random uniform positions in the serving and target cells in every Transmission Time Interval (TTI). The UEs' directional movements are selected randomly with a fixed speed throughout the simulation, which contains five different mobile speed scenarios $(30,60,90,120$, and $140 \mathrm{~km} /$ hour). The mobility movement of all users is considered to be inside the first 37 cells which are located in the close positions to the centre cell. Six eNBs are considered as the stations that cause the interference signals for each user during all the simulation time. The Frequency Reuse Factor (FRF) has been assumed to be one. Moreover, the Adaptive Modulation and Coding (AMC) scheme is considered based on the sets of Modulation Schemes (MS) and Coding Rate (CR) that were introduced in $[10,11]$. Handover procedure for LTE-Advanced system that was introduced in [12] is followed with assuming $6 \mathrm{~dB}$ as a handover margin level and 600 milliseconds as time-to-trigger (TTT). In addition, the Radio Link Failure (RLF) detection, Radio Resource Control (RRC) reestablishment procedure, and Nonaccess Stratum (NAS) recovery procedure are considered through the simulation in order to achieve high accuracy in the performance evaluation. The vital essential parameters used in this paper are considered based on the LTE-Advanced system profile that were defined by 3 GPP specifications in [10-13], as listed in Table 1. 


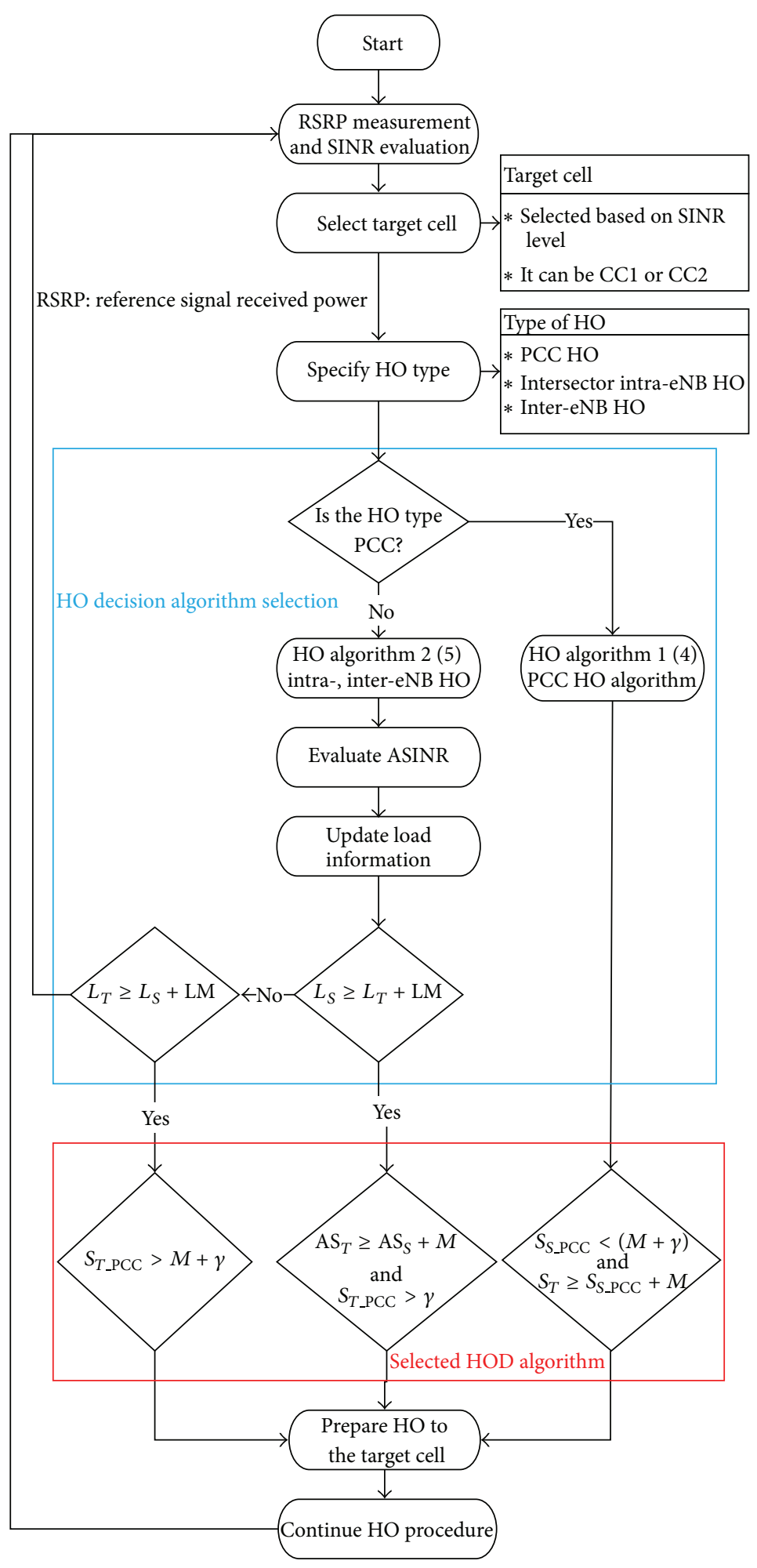

Figure 3: Flowchart of our proposed handover decision algorithm.

\section{Results and Discussions}

In this study, a simulation was used to validate the proposed HODA. The evaluation methodology of 3GPP LTE-Advanced system [10-13] is observed in the simulation as mentioned in Section 3. System performance evaluations achieved by MIF-AHODA and the other considered HODAs are presented in terms of user SINR, spectral efficiency, and user's outage probability as shown in Figures 5, 6, and 7, respectively. 


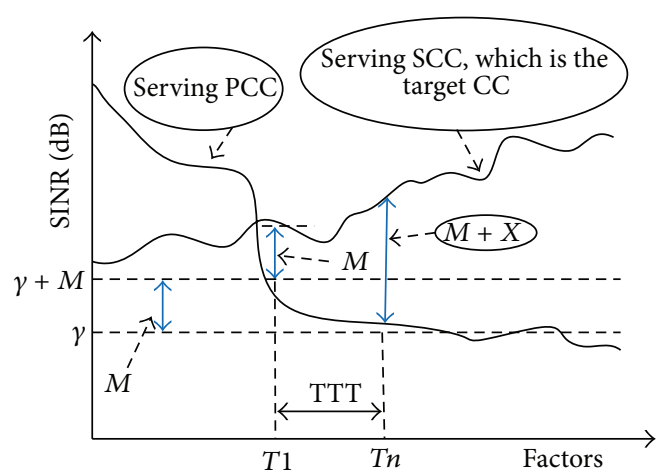

(a) HODA to switch PCC

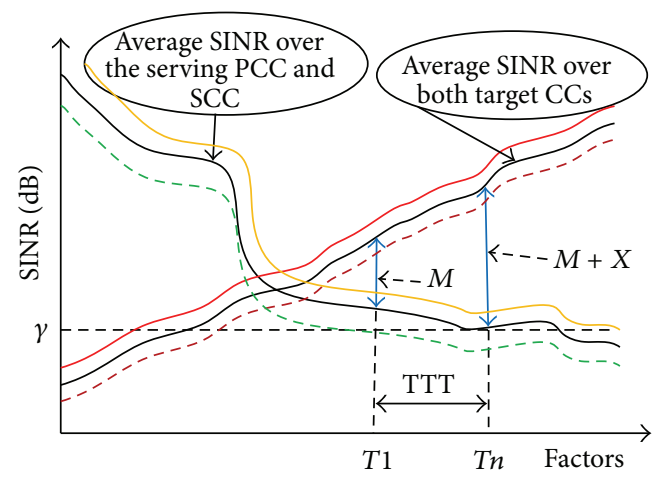

(b) HODA based on average SINR over both CCs

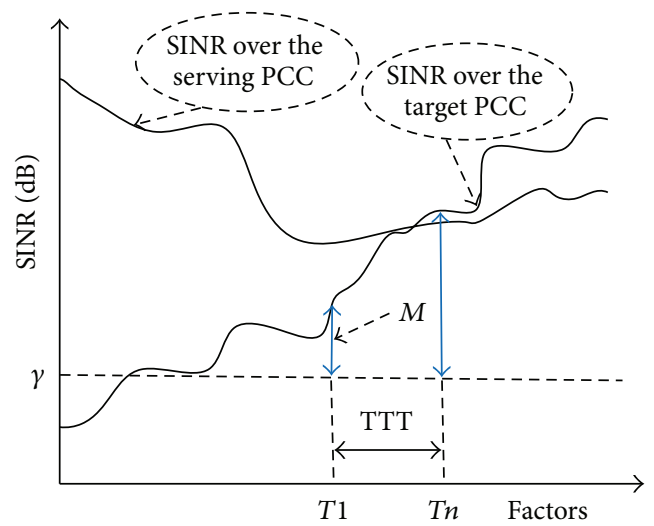

\begin{abstract}
$M$ : margin value $\gamma$ : threshold level of SINR TTT: time-to-trigger T1: the beginning of TTT $T n$ : the end of TTT

$X$ : greater or less than $M$
\end{abstract}

(c) HO decision based on resources availability

FIgure 4: Proposed handover decision algorithm description.

Figure 5 shows user SINR in dB based on different handover decision algorithms. The presented SINR represents the average users' SINR over the serving PCC, which is evaluated as the ratio of reference signal received power (RSRP) to the Interferences-plus-Noise-Ratio over each subcarrier assigned to the served user [14]. However, the results show that the MIF-AHODA enhanced user SINR by 13.5, 13.4, 3.45, and
TABLE 1: Simulation parameters [5-8].

\begin{tabular}{ll}
\hline Parameter & Assumption \\
\hline Cellular layout & $\begin{array}{l}\text { Hexagonal grid, 61 cell sites, } 3 \\
\text { sectors per cell site, 2 CCs per } \\
\text { sector }\end{array}$ \\
$\begin{array}{l}\text { Minimum distance between UE } \\
\text { and eNB }\end{array}$ & $\geq 35$ meters \\
Total eNB TX power & $46 \mathrm{dBm}$ per CC \\
Shadowing standard deviation & $8 \mathrm{~dB}$ \\
eNBs noise figure & $5 \mathrm{~dB}$ \\
UE noise figure & $9 \mathrm{~dB}$ \\
Operation carrier bandwidth & $20 \mathrm{MHz}$ (PCC and SCC) \\
Total system bandwidth & $40 \mathrm{MHz}(2 \mathrm{CCs} \times 20 \mathrm{MHz})$ \\
Number of PRBs/CCs & $100 \mathrm{PRB} / \mathrm{CC}$ \\
Number of subcarriers/RBs & $12 \mathrm{subcarriers} \mathrm{per} \mathrm{RB}$ \\
Number of OFDM symbols per & 7 \\
subframe & $15 \mathrm{kHz}$ \\
Subcarrier spacing & $180 \mathrm{kHz}$ \\
Resource block bandwidth & $-101.5 \mathrm{dBm}$ \\
Q_rxlevmin & $50 \mathrm{~ms} \mathrm{for} \mathrm{PCC} \mathrm{and} \mathrm{SCC}$ \\
Measurement interval & $10 \mathrm{~ms}$ \\
Each X2-interface delay & $10 \mathrm{~ms}$ \\
Each eNB process delay & $10 \mathrm{~s}$ \\
T311 & \\
\hline
\end{tabular}

$3 \mathrm{~dB}$ better than the HODAs in the literature which were taken as a base: RSS, RSS-D, SINR, and IINR, respectively.

Figure 6 shows a cell-edge user spectral efficiency based on different HODAs. The cell-edge user spectral efficiency is defined as the lower $5 \%$ of the evaluated throughput [bps/Hz] that can be received by the user $[13,14]$. However, the presented results show that MIF-AHODA achieves around 79.7, $80.7,12.7$, and $10.7 \%$ as average enhancement gains of celledge user spectral efficiency over HODAs based on RSS, RSSD, SINR, and IINR, respectively.

Figure 7 shows the user's outage probabilities that resulted from the simulation based on different HODAs. The user's outage probability $P\left(\operatorname{SINR}_{\mathrm{SPCC}}<\gamma\right)$ is recorded when the user's SINR over the serving PCC $\left(\right.$ SINR $\left._{S_{-} \text {PCC }}\right)$ falls below the threshold level, $(\gamma)$ [15], whereas the quality of service becomes unacceptable when SINR $_{S_{\text {_PCC }}}$ falls below threshold level. However, Figure 7 shows that MIF-AHODA reduces the user's outage probability by around $80,70,30$, and $25 \%$ as average reduction gains less than that resulting from HODAs based on RSS, RSS-D, SINR, and IINR, respectively.

The enhancements achieved by MIF-AHODA are due to the consideration of multiple influence factors and the optimal proposed algorithm that adaptively selects the suitable handover decision algorithm based on the handover scenario type and resource availability.

In case of a handover scenario type targeting switching the PCC, the handover decision is taken based on SINR 


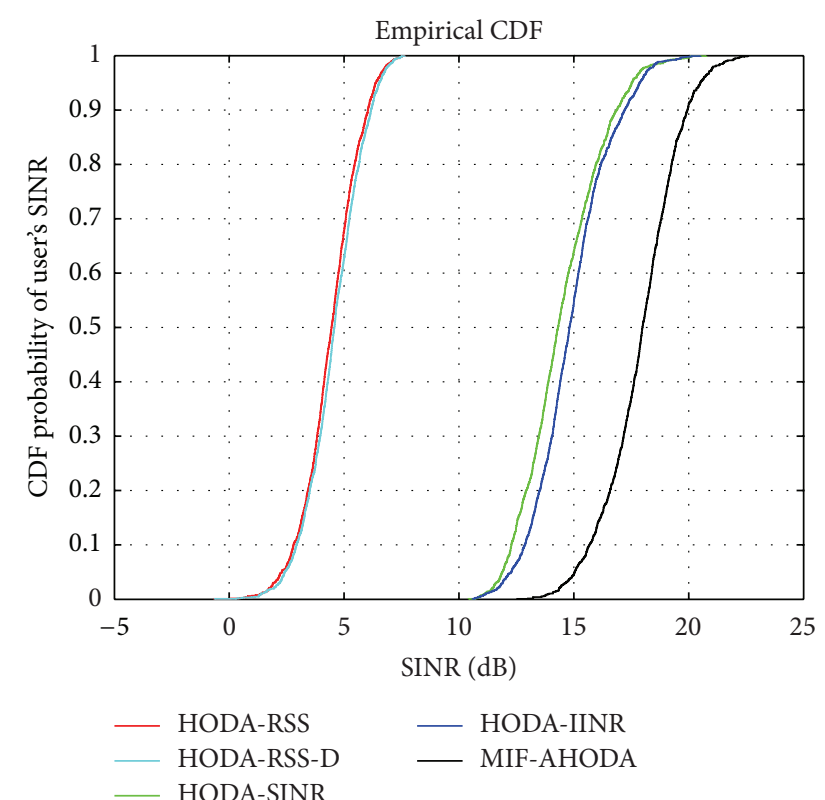

FIgURE 5: Average user's serving SINR over the PCC.

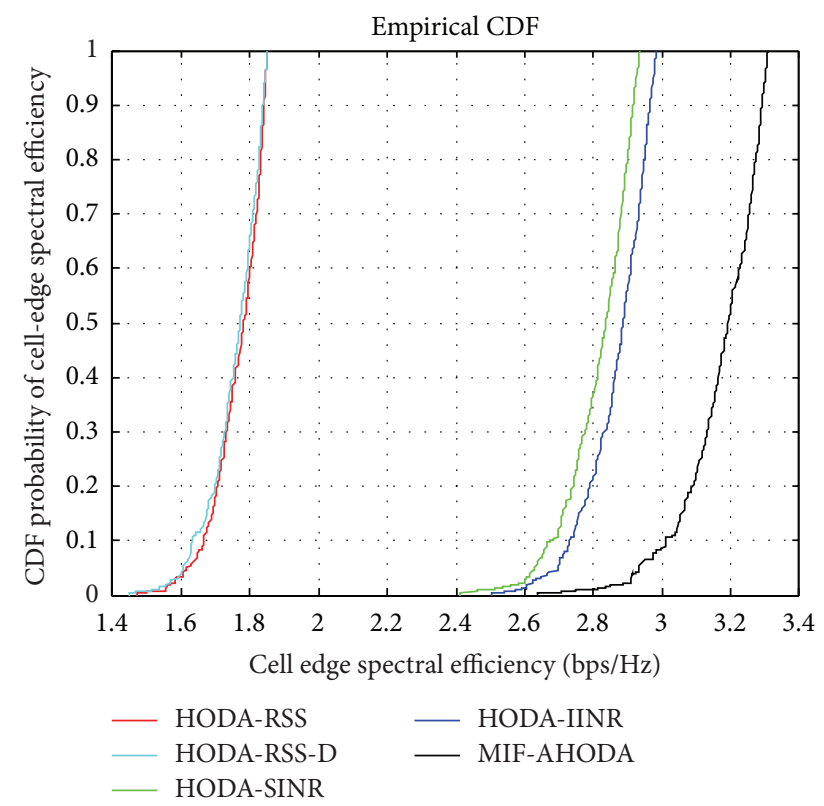

FIGURE 6: Average user's cell-edge spectral efficiency.

with hysteresis and threshold levels $\left(\operatorname{SINR}_{S}>M+\gamma\right)$. This algorithm takes true handover decision when the SINR over the serving PCC falls below the threshold plus margin level, as was illustrated in Figure 4(a) and expression (4). This then allows prevention of unnecessary handover procedure that can be performed between the PCC and SCC as long as the SINR over the PCC is greater than the threshold by margin level. Furthermore, this algorithm taking a handover decision before the signal over the serving PCC falls below the threshold level. That leads to decreasing user's throughput degradation and it contributes to avoiding the disconnection

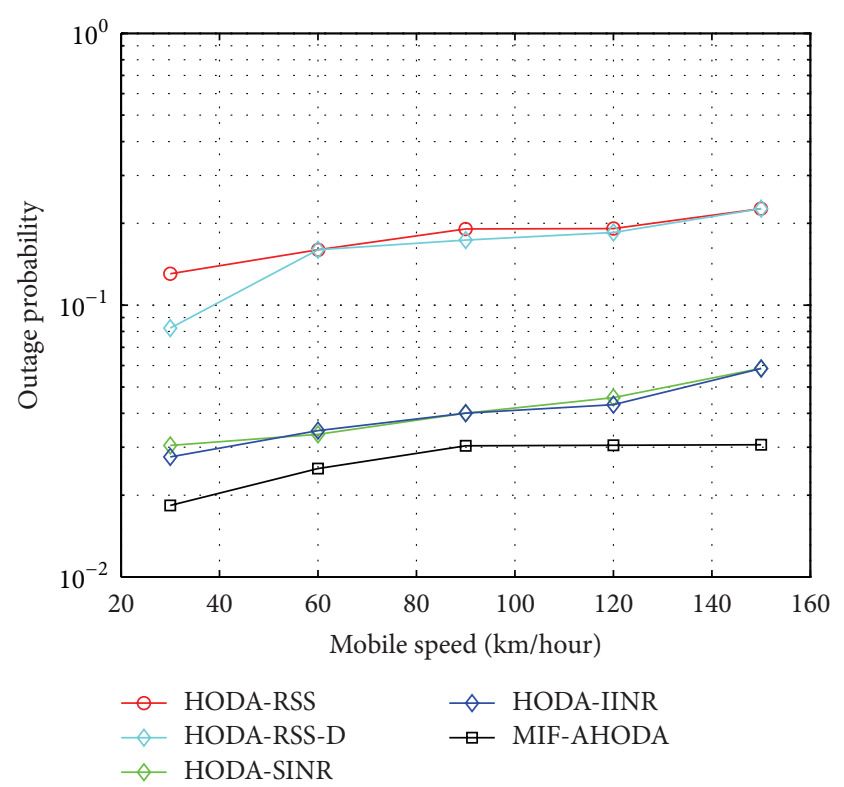

FIGURE 7: User's outage probability.

probability, which in turn leads to reducing user's outage probability.

In case a handover scenario type is targeting switching a user's connection to a new sector or new eNB, the handover decision can be adaptively taken based on two different algorithms, which are selected based on the resource availability as illustrated in Figures 4(b) and 4(c) and expression (5). If the resource availability of the serving cell $\left(L_{S}\right)$ is more than the target cell $\left(L_{T}\right)$ by resource margin level (LM), handover decision can be taken based on the average SINR over both PCC and SCC $\left(\mathrm{AS}_{T}>\mathrm{AS}_{S}+M_{\text {SINR }}\right)$. This leads to performing the handover procedure to the best target eNB and can provide better signal quality over both CCs, which in turn leads to providing more resources to the served user during the active mode time. That enhances user throughput and reduces outage probability. On the other hand, if the resource availability of the target cell $\left(L_{T}\right)$ becomes more than the serving cell $\left(L_{S}\right)$ by resource margin level (LM), handover decision can be taken based on the SINR over the target PCC only SINR $_{T_{\text {_PCC }}}>M+\gamma$. This leads to performing an early handover procedure to the target cell that has more resources. That leads to assigning more resources to the served user with acceptable signal quality, which in turn leads to enhanced user throughput and reduces outage probability.

\section{Conclusion}

It may be concluded that the proposed MIF-AHODA is a useful algorithm through the implementation of CA technology in LTE-Advanced system. It contributes to enhanced system performance from the perspective of user SINR, spectral efficiency, and reducing the user's outage probability. It is notably enhanced over the legacy RSS HODA, HODARSS-D, HODA-SINR, and HODA-IINR. Consequently, the 
proposed MIF-AHODA can be considered as one of the significant handover decision algorithms options which can be implemented in LTE-Advanced system.

\section{Conflict of Interests}

The authors declare that there is no conflict of interests regarding the publication of this paper.

\section{Acknowledgments}

The authors would like to acknowledge the financial contribution from Grant reference no. GUP-2012-036 for the publication of this work. This work was supported by the Universiti Kebangsaan Malaysia under Grant nos. GUP-2012036 and 01-01-02-SF0789 (MOSTI).

\section{References}

[1] G. P. Pollini, "Trends in Handover design," IEEE Communications Magazine, vol. 34, no. 3, pp. 82-90, 1996.

[2] K.-I. Itoh, S. Watanabe, J.-S. Shih, and T. Sato, "Performance of handoff algorithm based on distance and RSSI measurements," IEEE Transactions on Vehicular Technology, vol. 51, no. 6, pp. 1460-1468, 2002.

[3] K. Yang, I. Gondal, B. Qiu, and L. S. Dooley, "Combined SINR based vertical handoff algorithm for next generation heterogeneous wireless networks," in Proceedings of the 7th IEEE Global Telecommunications Conference (GLOBECOM '07), pp. 44834487, Washington, DC, USA, November 2007.

[4] H.-H. Choi, "An optimal handover decision for throughput enhancement," IEEE Communications Letters, vol. 14, no. 9, pp. 851-853, 2010.

[5] Y. D. Lee, S. D. Chun, S. J. Yi, S. J. Park, and S. H. Jung, "Carrier aggregation management method, system and devices," Google Patents US 2011/0028148 A1, 2011.

[6] P. J. Song and J. Shin, "Method for handover in multi-carrier system,” Google Patents, US20110070880, 2014.

[7] Z. Shen, A. Papasakellariou, J. Montojo, D. Gerstenberger, and F. Xu, "Overview of 3GPP LTE-advanced carrier aggregation for $4 \mathrm{G}$ wireless communications," IEEE Communications Magazine, vol. 50, no. 2, pp. 122-130, 2012.

[8] M. Iwamura, K. Etemad, M.-H. Fong, R. Nory, and R. Love, "Carrier aggregation framework in 3GPP LTE-advanced," IEEE Communications Magazine, vol. 48, no. 8, pp. 60-67, 2010.

[9] S. Jung, J. Kim, E. Kim et al., "Handover method in wireless communication system," Patent US20120113943 A1, 2012.

[10] 3 GPP, "E-UTRA; Radio Frequency (RF) system scenarios (Release 10) TR 36.942 V10.3.0," http://www.3gpp.org/.

[11] 3GPP, "Physical channels and modulation (Release 11)," Evolved Universal Terrestrial Radio Access (E-UTRA) TS 36.211, version 11.5.0, 2013, http://www.3gpp.org.

[12] 3GPP, E-UTRAN; Overall description (Release 11), TS 36.300 V11.9.0, France, 2012, http://www.3gpp.org/.

[13] E. Dahlman, S. Parkvall, and J. Sköld, 4G: LTE/LTE-Advanced for Mobile Broadband, Academic Press, New York, NY, USA, 2011.
[14] I. Shayea, M. Ismail, and R. Nordin, "Downlink spectral efficiency evaluation with carrier aggregation in LTE-advanced system employing adaptive modulation and coding schemes," in Proceedings of the IEEE 11th Malaysia International Conference on Communications (MICC 2013), pp. 98-103, IEEE, Kuala Lampur, Malaysia, November 2013.

[15] J. Paris and D. Morales-Jiménez, "Outage probability analysis for Nakagami-q (Hoyt) fading channels under rayleigh interference," IEEE Transactions on Wireless Communications, vol. 9, no. 4, pp. 1272-1276, 2010. 

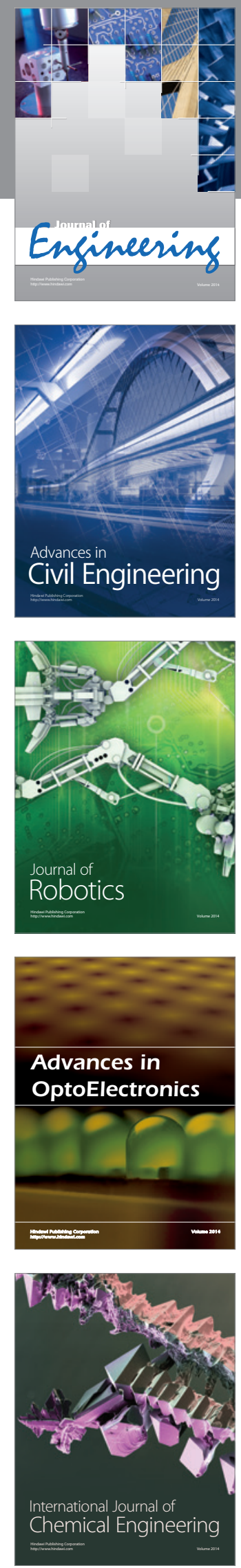

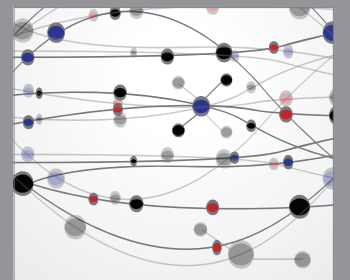

The Scientific World Journal
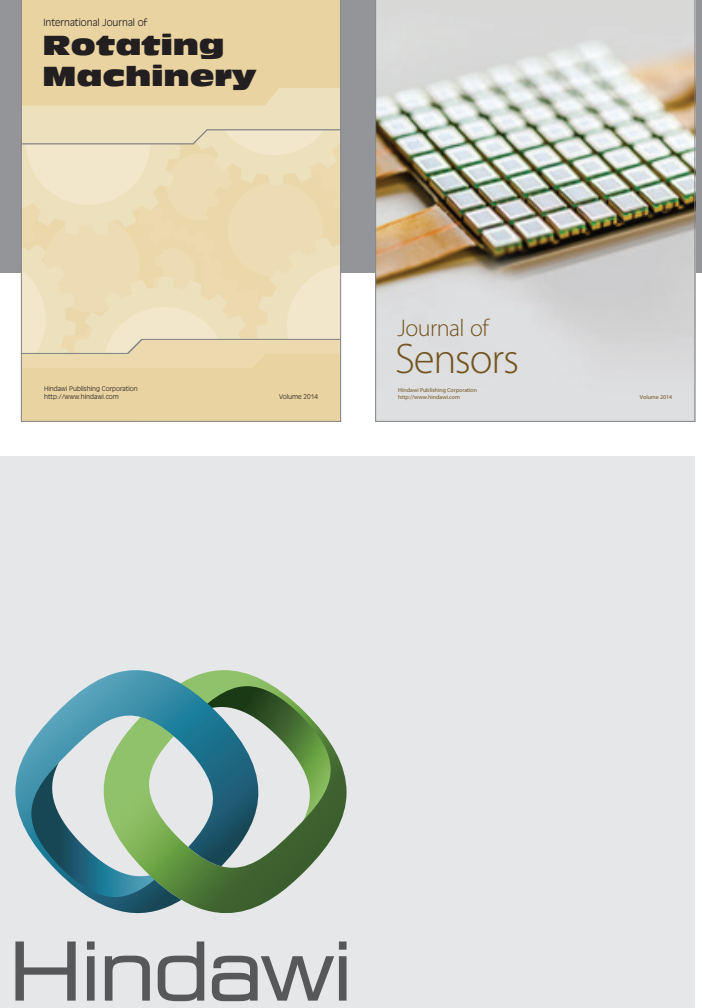

Submit your manuscripts at http://www.hindawi.com
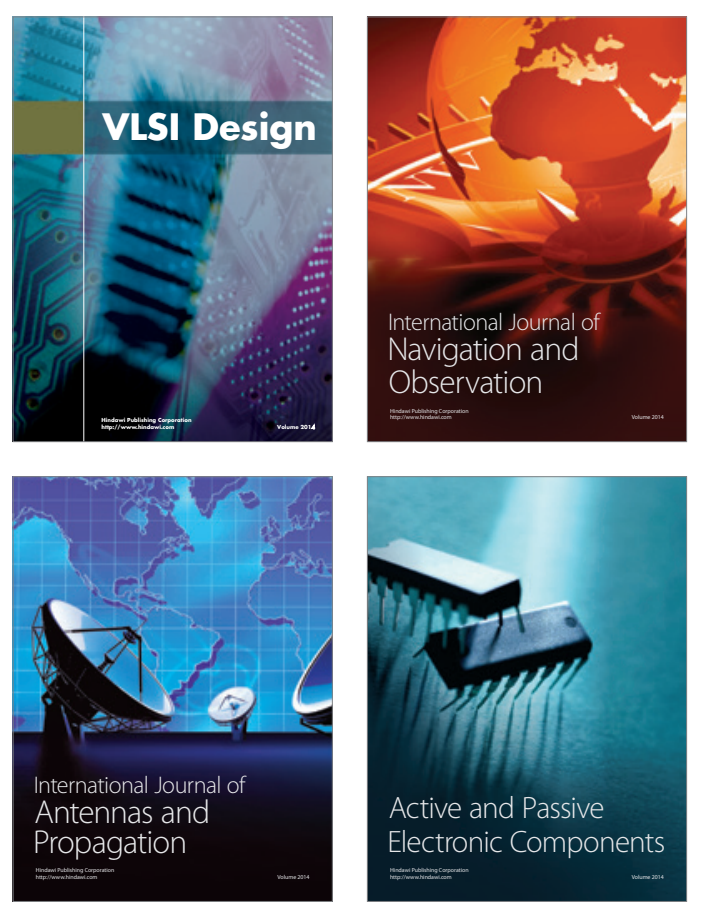
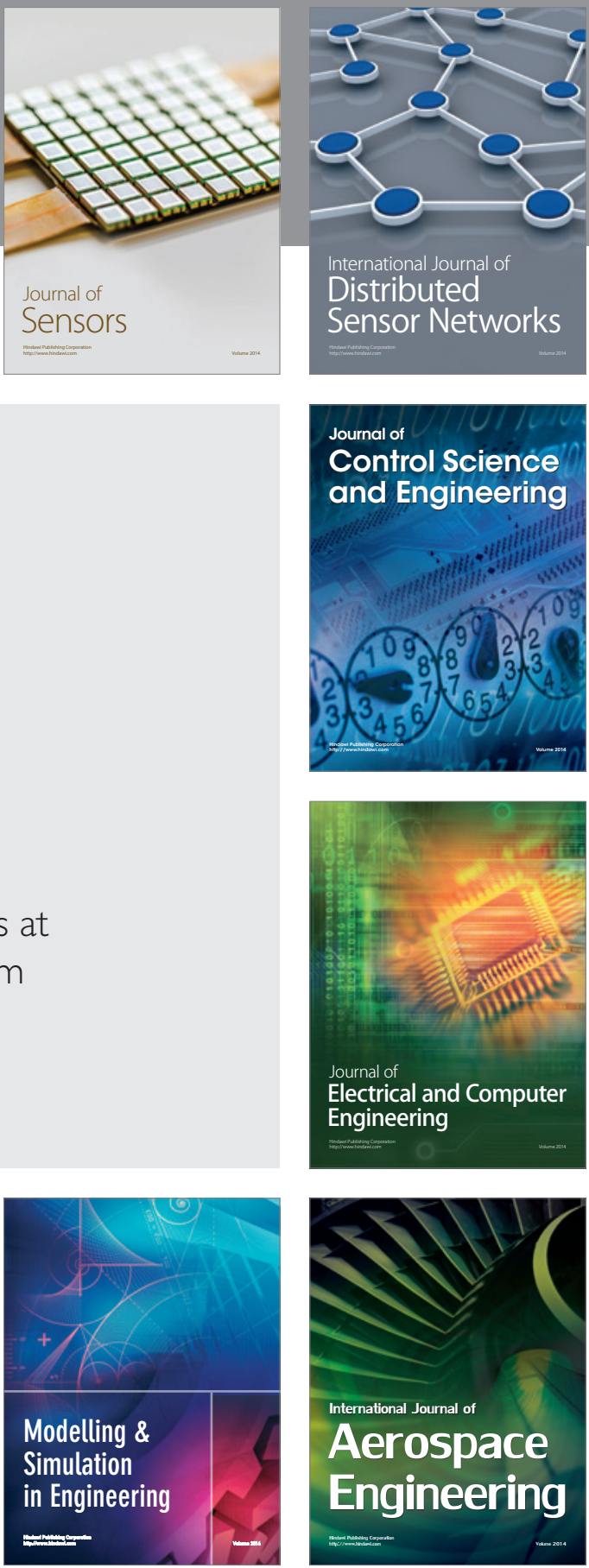

Journal of

Control Science

and Engineering
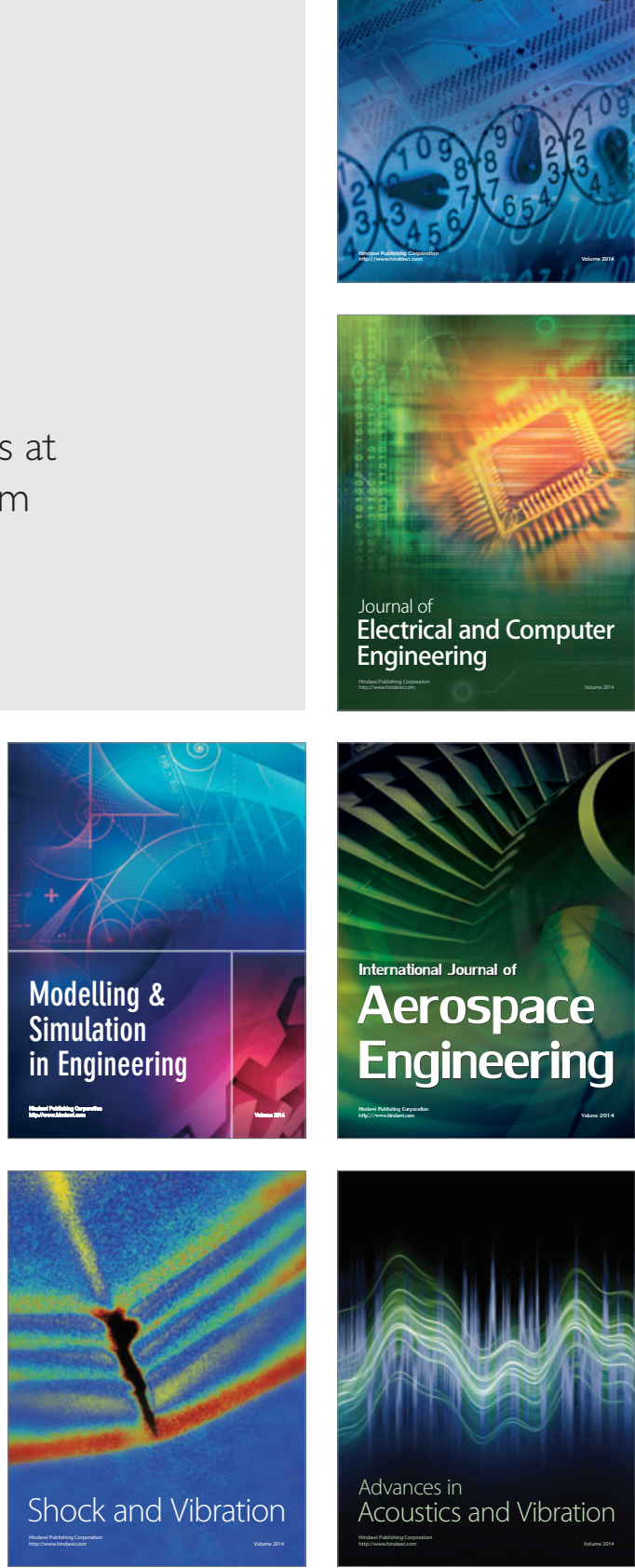